\title{
Asentamientos caxcanes en el Cañón de Juchipila y el primer intento del Camino Real de Guadalajara a Zacatecas
}

\author{
M. Nicolás Caretta* \\ UNIVERSIDAD AUTÓNOMA DE SAN LUIS POTOSÍ
}

En este trabajo se presentarán algunos elementos para identificar la primera ruta o camino que se quiso establecer como Camino Real que llevó a las minas de plata de Zacatecas, basado en la identificación arqueológica de sitios en el Cañón de Juchipila y la información que proporcionan las primeras fuentes que dan cuenta de las rutas usadas por los colonos conquistadores. A su vez, en este documento, se busca explorar los conflictos provocados entre los caxcanes y los espańoles y sus aliados que, finalmente, culminaron con la guerra del Mixtón y lo que pasó a ser la ruta definitiva del Camino Real de Guadalajara a Zacatecas. ${ }^{1}$

(Caxcanes, Camino Real, Cañón de Juchipila, Guadalajara, Zacatecas)

\section{INTRODUCCIÓN}

T

na vez realizada la conquista del Señorío Mexica, la destrucción de México-Tenochtitlan fue inevitable y de ella surgiría la capital de la Nueva España. Así, inmediatamente después de la caída del Imperio mexica los conquistadores continuaron con las exploraciones en búsqueda de tesoros, la extensión de sus territorios, el inventario de sus nuevas propiedades y con la cacería de almas para salvarlas de la perdición (si es que los nativos tenían, en absoluto, alma). De esta forma, las exploraciones en todas las direcciones y

*mictlan@rocketmail.com

${ }^{1}$ Quisiera agradecer al arqueólogo Sergio Ulloa por permitirme dar uso de la información de sus investigaciones del Camino Real de Guadalajara a Zacatecas. 
la conquista de estos aspectos y de sus habitantes no demoraron su inicio. Era necesario poblar nuevos territorios y ciudades para poder salvaguardar sus nuevas propiedades y organizar expediciones hacia el norte donde las míticas ciudades de Cíbola se encontraban.

Guadalajara estaba entre esas nuevas ciudades y se convirtió en un punto importante una vez que se localizaron las minas de plata de Zacatecas, las cuales fueron abiertas en 1546. A partir de ese momento, la avaricia de la Corona española, y del virrey, puso toda su atención en la industria de la minería, por tanto, la construcción de caminos entre las minas y la capital de la Nueva Espańa fue un elemento prioritario porque de éste dependía la explotación y entrega efectiva de productos.

A mediados del siglo xvi, Guadalajara se había convertido en la capital de la Nueva Galicia (de la cual también Zacatecas formaba parte) y necesitaba un camino que habilitara al gobierno local para comunicar y controlar a las ciudades bajo su jurisdicción. Pero la existencia de estas dos ciudades (Guadalajara y Zacatecas) ubicadas tan lejos de la capital de la Nueva España creó huecos territoriales donde ninguna población española vivía, permitiendo el desarrollo de grupos de resistencia entre las poblaciones indígenas locales. Esta situación fue la que se enfrentó en la región del Cañón de Juchipila, la ruta más directa entre Guadalajara y Zacatecas y su primer intento de Camino Real.

\section{Los CAMINOS}

Hasta cierto grado, todos los caminos usados por los españoles en su paso al norte, no únicamente el Camino Real, siguieron rutas ya establecidas por los nativos, las cuales cruzaban en todas las direcciones del territorio. De hecho, tampoco hubo una ruta única que siguiera en dirección al hoy suroeste de los Estados Unidos y que lo uniera con el resto de Mesoamérica. Se puede asegurar que, previo a la conformación de estos "caminos formales" que establecieron los conquistadores, estuvieron las rutas que las culturas locales ya tenían bien establecidas, de las cuales algunas fueron reutilizadas (Nicolás Caretta 2003). 
Desde la perspectiva general, Medina (2000) señala que los arqueólogos que han trabajado en este tema han dividido las antiguas rutas de comunicación prehispánicas como un sistema de veredas (o vías de acceso) y caminos, haciendo distinción entre ellos basándose en su anchura y en su grado de construcción. Las "veredas" son los caminos informales y los formales son los "caminos". ${ }^{2}$ En este sentido, los estudios arqueológicos han señalado que los caminos formales, en tiempos precolombinos, combinaron funciones de tipo económico, sociopolítico, integrativo, militar y religioso. No entraré en detalle sobre este punto ya que ha sido tratado por otros investigadores (idem), así que pasaré al siguiente punto.

Lo que sí es importante recordar es que los caminos no fueron únicamente el resultado del interés por la minería por parte de la Corona. Estos también fueron hechos con el fin de:

- Conocer al máximo la extensión de su nuevo territorio.

- La posibilidad de hacer un inventario de sus nuevas posesiones.

- La conquista religiosa.

- Facilitar la guerra contra los "salvajes" pueblos del norte.

- Extender los dominios de la jurisdicción de la Nueva España y la Nueva Galicia.

\footnotetext{
${ }^{2}$ Medina González aclara que "Las veredas (path) o caminos informales, se definen como aquellos senderos estrechos que sirven como canales de circulación peatonal entre puntos que se hayan geográficamente separados. Entre sus principales características se encuentran que son el producto del tránsito constante de animales y de personas, su ancho, aunque no muy amplio depende usualmente de las facilidades que otorgue a la circulación peatonal; en general, tienden a ser muy irregulares en la medida que evitan los obstáculos naturales que se encuentran durante su recorrido y muestran varias rutas alternativas para dirigirse a un mismo lugar. La redundancia en sus conexiones se debe a las necesidades inmediatas, usos, costumbres en el nivel grupal o de unos cuantos individuos y a la falta de planeación para su establecimiento. La poca inversión de fuerza de trabajo que se requirió para su creación estuvo dirigida a la limpieza de los materiales que se localizaban a todo su largo, a la estabilización o nivelación menor de las superficies de transporte que, en la mayoría de los casos, coinciden con la topografía natural del terreno. Los caminos formales se definen, por toda la evidencia arqueológica relacionada con aquellas rutas de transporte que fueron sometidas a la construcción deliberada, para servir como canales de comunicación, entre asentamientos arqueológicos, áreas de actividad, producción, distribución y consumo. En la mayoría de los casos, su formalización hizo necesaria la intervención de la construcción y el manejo de la ingeniería" (Medina 2000, 9-10).
} 
- Servir como referentes geográficos para el reparto de tierras en una escala menor, para el otorgamiento de tierras por gracia de la Corona a sus nuevos colonos y se convirtieron en la columna vertebral de los primeros asentamientos españoles en los nuevos territorios.

- $\mathrm{Al}$ interior de los asentamientos, los caminos se convirtieron en el eje desde el cual las calles eran planeadas, se estableció la traza urbana y se dividieron los lotes que serían otorgados a los conquistadores más prominentes.

- En algunos casos, los caminos marcaban las divisiones entre los barrios de indios y el barrio de espańoles, estableciendo en su camino la división jerárquica en el uso de los espacios. Es importante señalar que aun cuando los caminos fueron fronteras físicas entre la propiedad privada y la pública, éstas no lo eran siempre para la relación social.

Pero, en una mayor escala, los caminos funcionaron como fronteras, al igual que como axis de la estrategia colonial para conectar puntos geográficos entre el centro (capital) y el resto del territorio, dividiéndolo en fracciones más pequeñas que supuestamente permitirían mejor control sobre ellas (el cual falló como se mostró a la postre).

Otros caminos, como fueron los casos de Pánuco-México y Compostela-México, son claros ejemplos de esta relación, ya que ellos no solamente conectaban a la capital de la Nueva España, sino también aseguraban el territorio. Estos caminos fueron utilizados para establecer los límites del continente tanto al interior como al exterior al demarcar la línea costera del Golfo de México y del océano Pacífico. Otro caso similar fue el del camino que conectaba a Guadalajara con Tlaltenango y Nochistlán, el cual demarcaba el avance de los conquistadores en el territorio que pertenecía a los caxcanes (Baus 1982).

Hubo otros, como el Camino Real de México-Zacatecas y el Camino de Guadalajara a Zacatecas, que dividieron el territorio para minimizar las áreas inexploradas que quedaron entre la capital de la Nueva Espańa y Zacatecas durante el auge de la explotación minera. 
Durante el siglo xvı, la Nueva Galicia y el norte de la Nueva España se fueron llenando de caminos que sirvieron para mejorar e incrementar el flujo de soldados, colonos, trabajadores, productos e impuestos. Ellos tuvieron un papel importante en la economía local y extraterritorial estableciendo lugares, rutas e itinerarios fijos para el transporte (Calvo 1997). Estas razones, entre otras tantas, impulsaron a los conquistadores a buscar y crear más y nuevas rutas hacia el norte.

Para mediados y finales del siglo xvI existían ya tres rutas para comunicar a Guadalajara con Zacatecas, y era el camino que iba a través de la región de los Altos de Jalisco y los llanos de Aguascalientes el que se consideraba la última opción ya que era el más largo, aunque, al mismo tiempo, ofrecía las mejores condiciones; era el más fácil de resguardar y con él se evitaba ir a través de las sierras. Sin embargo, es importante notar que, a pesar de estas condiciones, esta ruta no fue duradera y algunos factores históricos a fines del siglo XVI crearon nuevas condiciones que hicieron necesario su uso parcial y la creación de nuevas rutas.

\section{El trazo del Camino Real de Guadalajara a Zacatecas}

De Guadalajara a Zacatecas.

[...] la ciudad de Guadalax[ar]a, que es la principal deste reino y donde reside la real Audi[enci] a y hay iglesia catedral, dista deste pu[ebl] o veintiuna leguas y, las mi[n]as de los Zacatecas, q[ue e]stán pobladas de españoles, veintiséis leguas. Y este pu[ebl]o está en medio de la d[ic]ha ciudad y minas, porque [e]stá [en] el camino real que, de la una p[ar]te, va a la otra.

[...] el camino que hay deste pu[ebl] o a la ciudad de Guadalaxara, es llano más que montuoso, excepto que, dos leguas cerca de la d[ic]ha ciudad, está una barranca grande, q[ue] tendrá una legua de bajada y otra de subida, y, por medio della, pasa un río caudaloso q[ue] llaman el Río Grande. $\mathrm{Y}$, deste pueblo a las $\mathrm{d}[\mathrm{ic}]$ as mi[n]as de los Zacatecas, es toda tierra muy llana, que se camina de ordinario en carretas y harrias; aunq[ue], toda ella, peligrosa, de guerra de chichimecas; $y$, cuando se camina, es con escolta de soldados españoles q[ue e]stán en presidios por man[da]do de su Maj[esta] d. Y [en] el d[ic]ho camino, [a] ocho leguas deste pu[ebl]o, está poblada una villa q[ue] llaman $\mathrm{N}$ [uest] tra S[eñor]a de la As[u]nción, donde está un 
fuerte q[ue] llaman Aguascalientes (Hernando Gallegos, Relación del pueblo de Teucaltiche, 1584, en Relaciones geográficas del siglo XVI, René Acuña, 1988, 302-3).

No está de más señalar que el camino que ha sido mencionado, tenía como objetivo unir la población de Guadalajara con las minas de Zacatecas. La descripción hecha del camino de Teocaltiche a Guadalajara fue lo que permitió a Ulloa (2002) inferir que se había hecho referencia a los caminos que iban a través de la región de Los Altos y no su predecesor en la Sierra de Nochistlán, al igual que la barranca mencionada es la que se forma por el Río Grande o Santiago, el cual al mismo tiempo rodea la ciudad de Guadalajara. El camino de Teocaltiche a Zacatecas era apto para el transito de carretas y recuas y llevaba al Presidio de Aguascalientes evadiendo la Sierra del Laurel y desde allá seguía las planicies que van de Aguascalientes a Guadalajara.

Ahora bien, el hecho de que la ruta a Zacatecas pasara a través de Teocaltiche suena lógico si consideramos que el asentamiento tuvo un papel importante en la colonización de Los Altos. Después de que los españoles pasaron penosas experiencias en territorio Caxcán y enfrentaron muchos problemas para establecer asentamientos en la región de los cañones, en particular el Cañón de Juchipila como lo veremos más adelante, Teocaltiche se convirtió en la opción más viable, ante todo porque los conflictos y las epidemias estaban causando estragos en toda la región y se volvió imposible para los nativos resistir la ocupación española en sus tierras. Posteriormente, porque la situación geográfica de Teocaltiche permitió a los españoles un desarrollo productivo y, por tanto, el asentamiento ganó importancia en el extremo de la Nueva Galicia. Desde este lugar, nuevos asentamientos empujaron las rutas hacia el norte. Algunos de los lugares que permanecieron son: Santa María de Los Lagos y Nuestra Señora de San Juan. La extensión del territorio que permaneció dentro de la jurisdicción de Teocaltiche durante el siglo Xvi señala esta importancia. Teocaltiche al igual que Aguascalientes y Lagos fueron parte de una estrategia de defensa colonial en contra de los grupos chichimecas (fuesen los caxcanes, guachichiles u otros). 
¿Durante cuánto tiempo estuvo esta ruta resguardada? Es difícil de tener una respuesta clara para esta pregunta, se asume que un factor importante yace en la culminación de la Guerra Chichimeca en 1600, razón por la cual la ruta de México-Zacatecas vía León, Lagos y Aguascalientes tomó más importancia y, por tanto, la conexión Guadalajara-Lagos que iba a través de Los Altos de Jalisco se sobrepuso a la de Teocaltiche. En el siglo xvir, la villa de Santa María de los Lagos se convirtió en un centro religioso debido a su locación como unión entre estos dos caminos, entonces Teocaltiche tomó un rol secundario, esta situación prevaleció al menos hasta el siglo XVIII cuando la industria de la minería decayó en Zacatecas.

Después de que el apogeo de la minería de Zacatecas decayera hasta su punto más bajo, el tráfico comercial disminuyó y la ruta a Guadalajara tomó otro camino, sin embargo, el camino anterior no fue nunca abandonado del todo pues asumió un uso interregional, aunque éste nunca más fue usado de la forma que fue concebido originalmente. ${ }^{3}$

\section{Los ASENTAMientos PREHISPÁNicos EN EL CAÑóN DE JUCHIPILA}

No debería sorprendernos que un gran número de sitios estuvieran situados en esta área y que el paso de los nuevos colonos hacía las minas de Zacatecas causara conflicto con los habitantes originales de la región. Estas circunstancias, como se ha señalado, son las que

${ }^{3}$ Para los propósitos de identificación de estos puntos en un mapa, Ulloa (2002) consideró necesario hacer una primera aproximación al origen y antigüedad de los asentamientos actuales para su mapa enfatizando la posibilidad de que todas las poblaciones hubieran cambiado sus nombres originales y, por tanto, se dificultara la tarea de aclarar el origen prehispánico de sitios, los asentamientos refundados por los españoles y los asentamientos españoles originales. Este mismo investigador menciona al menos tres tipos de denominaciones que fueron detectados: a) Aquellos que provienen del náhuatl o alguna otra lengua local y el tiempo que han estado expuestos a modificaciones y adaptaciones al castellano (Mitic, Tepantitlan, Acatic, Teocaltiche, etcétera); b) Aquellos que tienen el nombre de las funciones que solían tener (El Refugio, El Paso, La Estancia, La Labor, El Molino, El Sitio, La Ciénega, La Hacienda, El Astillero, etcétera); c) Aquellos que son resultados de eventos históricos (Villa Hidalgo, Arteaga, José Ma. Morelos, 20 de noviembre, etcétera). 
forzarían que posteriormente se cambiara la ruta de abastecimiento y distribución de recursos entre Guadalajara y Zacatecas por la vía Teocaltiche-Aguascalientes y con ello la aparición de nuevos asentamientos coloniales.

En los últimos años, los trabajos de investigación en el noroeste de la Mesoamérica mayor han cambiado mucho la visión de la Mesoamérica marginal, la de los grupos puramente salvajes que vivían en perpetuo estado de precariedad, que no tenían ningún grado de integración y que su presencia no se podía rastrear más allá del Posclásico. Grupos alejados de todo y de todos, a los que siempre se les manejó en paquete con el nombre genérico de chichimecas (Nicolás Caretta y A. Motilla 2008).

Ahora que estas ideas empiezan a disiparse y a tomar nuevos rumbos, podemos revalorar en su justa dimensión lo que fue esta región de Mesoamérica y verla como una macrorregión integrada a esferas de interacción más dinámicas y menos rígidas, sitios de mayor tamaño, frecuencia y complejidad. De esta forma, cuando buscamos tratar de entender los procesos que se dieron en esta región, debemos considerar estas variables y con ellas intentar dar nuevas alternativas de explicación (idem).

Una de las tareas colaterales de investigación del Proyecto Arqueológico Las Ventanas ( PALV $^{4}$ ), municipio de Juchipila, Zacatecas, fue ubicar todos los sitios arqueológicos registrados en la región, ${ }^{5}$ tratando de diferenciarlos por medio de su cronología y tamaño a partir de los informes registro con los que cuenta el INAH. De igual forma, el paLv busca recrear las condiciones biogeográficas e históricas que se pudieron haber tenido en épocas pretéritas. Así, parte del trabajo ha sido la realización de una base de datos en sig con un número de variables consideradas como importantes que permitiera hacer una mejor interpretación de los posibles escenarios presentados en los niveles de región y macrorregionales. Entre estas variables

${ }^{4}$ El palv estuvo bajo responsabilidad directa de quien suscribe este texto hasta concluir la temporada 2004, posteriormente estaría bajo la responsabilidad del arqueólogo Armando Nicolau, quien ha mantenido su continuidad hasta la fecha.

${ }^{5}$ Lo que concentraría principalmente los sitios que ahora se encuentran dentro del actual estado de Zacatecas, Jalisco, Aguascalientes y San Luis Potosí. 
estuvo la identificación y la reconstrucción a través de datos históricos, etnohistóricos y arqueológicos de los posibles puntos donde estuvieron emplazados tanto las rutas naturales que se siguieron en el primer intento de Camino Real Guadalajara-Zacatecas, así como el segundo, además de sus asentamientos.

En el caso hipotético de la ruta prehispánica, se ha utilizado como base la información de sitios arqueológicos distribuidos por esta zona y registrados por el INAH en Jalisco y Zacatecas. Se consideró y revisó un número considerable de información bibliográfica, mapas, registros arqueológicos, fotos aéreas, imágenes de satélite, habitantes de la región y trabajo en campo. En mi caso, no era necesario encontrar información exacta del número preciso de jornadas que se hubieran podido emplazar a lo largo del Cañón de Juchipila y la relación con las poblaciones precolombinas. Así que sólo me dediqué a buscar los sitios que se encontraban dentro del cañón y del valle desde la parte sur que va del ahora estado de Jalisco al de Zacatecas con dirección a la zona minera. Muchos, si no es que todos estos asentamientos precolombinos, ahora se encuentran cercanos a las poblaciones novohispanas establecidas una vez que se realizó la conquista de la región.

Es importante señalar que no se tiene información precisa acerca de todos estos sitios y sus fechamientos tampoco son claros, aunque si consideramos los materiales que fueron observados en superficie por los diversos proyectos que describen esos sitios podemos considerar cierta afinidad cultural con otros identificados y trabajados en diversos puntos, particularmente aquellos pocos que sí cuentan con fechas radiocarbónicas. Muchos elementos corresponden por lo menos al Epiclásico y Posclásico, lo que para fines de este estudio es suficiente.

Por lo que se puede apreciar en los restos arqueológicos presentes y por los datos históricos que se cuentan para esa zona se puede considerar una extensión temporal y espacial bastante extensa. Juchipila o Xuchupila aparece también como Taltan en las fuentes (Tello 1984, IV, 47 [1542]), población que fue conquistada por los caxcanes en el siglo XII (??) (Baus 1985, 97) y estuvo bajo su control hasta la entrada de los españoles en el siglo Xvi (Weigand 1985). El Cerro de Las Ventanas es identificado por Weigand (1985, 169-170) 
como el "Peñol de Juchipila" y cercano al "Peñol del Mixton" de las Crónicas de la conquista del Reino de Nueva Galicia (1963, 343 [1542]), otro centro ceremonial y político de los caxcanes. Al parecer la lengua de los caxcanes era una variante del náhuatl similar al de los mexicas, el cual por la forma brusca de ser de los caxcanes y su manera de hablar se les refería como "mexicanos rústicos", "tochos" o "villanos" (Baus 1985, 98; Tello 1984, IV, 40). Inclusive Tello (1984, II, 19, 382), hace referencias muy claras de la relación existente entre los mexicas y los caxcanes no sólo como parte de un grupo lingüístico sino también como adoradores de, al menos, una deidad en común, Huitzilopochtli.

De la misma información que podemos encontrar en las fuentes y de los restos arqueológicos observados en superficie y análisis de materiales cerámicos se puede asumir una larga ocupación y desarrollo social en este sitio. ${ }^{6}$

Durante el diseño del North-Central Frontier of Mesoamerica, ${ }^{7}$ tanto el valle de Juchipila como el valle de Malpaso fueron definidos

${ }^{6}$ El Dr. Phil Weigand, afirma que cuando menos existen cuatro tipos cerámicos del Cerro de Las Ventanas y el valle de Juchipila (Weigand 1985, 173-175). El primero de estos tipos cerámicos, el llamado Policromo Negativo de Las Ventanas, según Weigand es una variación local (valle Juchipila) de los estilos que aparecen en la zona de Chupícuaro durante el Preclásico. Entre éstos se distinguen las figurillas, las vasijas trípodes, copas y escudillas. De igual forma, menciona que este tipo de material también se ha descubierto hacia el norte de esta región en el Valle de Mal Paso, usualmente asociado a entierros. El segundo tipo, Bicromo Negativo de Las Ventanas, se encuentra distribuido tanto en áreas de habitación como entierros. Entre los materiales característicos de este tipo se encuentran platos, jarros, escudillas, así como vasijas trípodes. Este tipo de material es identificado como el mismo que se ha recuperado en la región de Chalchihuites (llamado Negativo A), en el valle de Malpaso, en el Cañón de Bolaños y otras áreas de Jalisco. Weigand afirma que el tercer tipo de material (Policromo de Las Ventanas), también aparece en sitios preclásicos de Jalisco. Las formas usuales en que aparece este material cerámico son: jarros, platos, figurillas, escudillas y vasijas trípodes. Finalmente, el cuarto tipo cerámico, bautizado como Negro en Rojo de Las Ventanas, se encuentra frecuentemente en vasijas y figurillas. Este material se encuentra durante los periodos Clásico y Postclásico en la zona y por sus semejanzas podría estar asociado a materiales del Suroeste Estadounidense y los llamados Policromos de St. Johns y Wingate de acuerdo a Weigand.

${ }^{7}$ C. Kelley, W. Taylor y Pedro Armillas, Studies of the North-Central Frontier of Mesoamerica. Archaeological and Ecological Investigation of the Northe Central Frontier of Mesoamerica and the Relationships of the Cultures of Central Mesoamerica, the Gran Chichimeca, and the American Southwest, September 1, 1961-August 31, 1963 (2 years), ms., Submitted by the Southern Illinois University Carbondale, Illinois, pp. 1-37. 
como Área 4. La definición o, mejor dicho, la percepción que Kelley hizo de esta "área arqueológica" se basaba en correlaciones observadas en análisis detallados que hizo de la colección de materiales del Sr. Sescosse provenientes de la región de Apozol-La Purísima-La Tirisia en Juchipila y su comparación con otras colecciones.

De igual forma, Schöndube $(1980,173)$ había señalado que la tradición de tumbas de tiro del occidente mexicano y la tradición Chupícuaro se mostraban muy poco traslape, a excepción de la zona de Los Altos de Jalisco y Juchipila. Esta confluencia de Preclásico Tardío y Clásico Temprano se manifiesta en el complejo cerámico del valle de Juchipila. A la fecha, los materiales cerámicos identificados en este valle fueron vasijas del grupo Colorines descrita para el complejo Tabachines del valle de Atemajac, una región considerada dentro de la tradición de tumbas de tiro del occidente mexicano (Galván 1991, 48-50). En éstos se presentan cuencos trípodes y platos negativos polícromos de Apozol y Juchipila que son análogos a los que fueron encontrados en Cerro Encantado en la región vecina de Los Altos de Jalisco (Bell 1974). Éstos están directamente relacionados con los tipos identificados como componentes del complejo Morales del noroeste de Guanajuato (Braniff 1972, 1998; Jiménez 1988). Éstas son las cerámicas tempranas que Kelley definió como tipos de "filiación Chupícuaro". En el Cerro Encantado, estas cerámicas negativas diagnósticas se encontraron asociadas con las figurillas huecas conocidas como "cornudos", asociación que también se da en la zona de Apozol. De hecho, reciente trabajo de rescate hecho en Juchipila proporcionó materiales negativos pintados, ollas como las que fueron encontradas por Bell (1974, fig. 7) en Cerro Encantado.

Los nexos existentes con Colorines-Tabachines/Morales también han sido definidos en Los Altos de Jalisco como fase I (200 a. C. -300 d. C.) y parece indicar la integración del valle de Juchipila dentro de la región de Los Altos desde fechas muy tempranas. Pero sobre todo, esta convergencia se muestra por la presencia de trompetas de caracolas, espejos de pirita, figuras (huecas) de "cornudos", y diseños geométricos policromos sobre negativos, algo que puede ser considerado como del complejo Morales de Guanajuato. 
Un tercer componente debe ser considerado para la región para todo esto, una vez que Kelley analizó la colección de Sescosse y artefactos provenientes de Totoate en el valle de Bolaños. Los cuencos trípodes (garras o bulbos huecos) negativos pintados del valle de Juchipila tienen analogías directas con elementos recientemente encontrados en una tumba de tiro sellada en el valle de Bolaños, y la cual dio fecha radiocarbónica de entre los II y IV siglos d. C. (Cabrero y López 1988, figs. 19, 105, 108). Estos elementos culturales de Bolaños sugieren una confluencia dentro del valle de Juchipila de tres de los mayores sistemas culturales regionales del occidente de México. Es de suponerse que las dinámicas culturales resultantes de estas confluencias de tradiciones del occidente mexicano estuvieron relacionadas de alguna manera a los procesos que produjeron la oleada inicial de la expansión mesoamericana hacia el valle de Malpaso y la región de Chachihuites, que dieron como resultado le horizonte Canutillo-Malpaso.

Desde al menos la parte temprana del periodo Clásico Medio, las vasijas Negra Esgrafiada con Rojo son las cerámicas diagnósticas para el valle. Una variedad especial ha sido previamente identificada como una variante regional del Atoyac inciso de la cuenca de Sayula (Noyola 1994, fig. 5). De la misma manera, las excavaciones hechas en el Cerro de Tepisuazco, Jalpa, produjeron gran cantidad de este material. También las colecciones de Sesosse y Muro provenientes de Apozol incluían este tipo cerámico. La distribución de este material es continua desde el valle de Juchipila al valle de Atemajac (Galván 1976, láms. 11 y 13; Schöndube comunicación personal 1994), a la cuenca de Sayula y hasta la costa de Colima, en lo que Ramírez Urrea (1997) ha propuesto como un marcador cerámico de una esfera de interacción mayor que se dio del 500 al 750 d. C. El elemento diagnóstico que se dio a través de esta extensa esfera es la figurilla Clase F de Cerrito de García (Gómez Gastélum y de la Torre 1996, 142), esfera a la cual Jiménez y Darling $(2000,169)$ han llamado Jalisco-Colima. Estas figurillas de Cerrito de García están presentes en el valle de Juchipila, en las zonas de Apozol y Jalpa, y en el valle de Bolaños (Kelley y Hrdlicka encontraron este tipo de figurillas en su excavación en Totoate). Cabrero ha detectado este tipo de figurillas 
en sitio llamado en Piñón en el valle de Bolaños. La cronología para el sitio las figurillas F de Cerrito de García es de aproximadamente 600-900 d. C. (Ramírez Urrea 1997).

De la misma forma, por que se refiere a la cerámica negativa pintada de base anular del periodo Epiclásico constituye un importante marcador de este horizonte cultural. La distribución de este tipo de material cerámico diagnóstico se extiende desde el valle de Juchipila (Cerro de Tepisuazco, Jalpa y Apozol) hasta la región de Los Altos (López et al. 1994), hacia el este a León, Guanajuato (López y Ramos 1992), al norte a través de la región de Encarnación de Díaz (Jalisco y Aguascalientes) hasta la región de Villa García en la parte este de Zacatecas y el sur del valle de Atemajac (Schöndube y Galván 1978). Este tipo cerámico también ha sido encontrado como material intrusivo en la cuenca de Sayula (Noyola 1994, 79, fig. 4). La vasija negativa de base anular de Juchipila es una cerámica de elite, contemporánea a la cerámica negativa de Tepozan del complejo de La Quemada hacia el norte. Los dos tipos comparten los mismos diseños iconográficos, aunque este tipo de cerámica de base anular no ha sido identificada en el valle de Malpaso todavía. La distribución de la cerámica negativa pintada de base anular (cuencos) puede ser considerada como evidencia importante de la esfera de interacción que une a estas dos regiones contiguas, que tentativamente pueden ser identificadas como la subesfera Altos-Juchipila.

Fragmentos de secciones de moldes faciales de cerámica tipo efigie constituyen otro elemento diagnóstico del valle de Juchipila. Éstos han sido encontrados en San Aparicio en la región de Los Altos (Jiménez 1989, 14, fig. 2; 1995, fig. IB), en el valle de Atemajac (Schöndube y Galván 1978; Schöndube 1983), y en los sitios de Cerritos Colorados y Atoyac en la cuenca de Sayula (Noyola 1994, 62-3, figs. 2 y 3; Ramírez Urrea 1997).

Entre los componentes arquitectónicos del Epiclásico de Tepisuazco se han recuperado una amplia variedad de vasijas pseudocloisonné, al igual que ornamentos manufacturados en concha, piedras verdes y turquesa. Ya mencionamos anteriormente, una variedad específica de una vasija pseudocloisonné decorada y hallada en Tepisuazco, la cual es del mismo tipo encontrado en La Quemada y Totoate. 
Jiménez Betts detectó en 1991 un complejo circular Guachimonton en el sitio del Cerro de Tepisuazco, donde el patrón de la arquitectura predominante es de un complejo pirámide-plaza de tipo rectangular. Desde entonces éste ha sido verificado en varias ocasiones por Weigand (Weigand et al. 1999). La presencia de arquitectura de plazas rectangulares con arquitectura circular también ocurre en Totoate (Kelley 1971). A la fecha, la cronología de las estructuras principales sólo es tentativa. La presencia de cerámica Gavilán Policromo y Amapa (Meighan 1976), en Tepisuazco, podría relacionarse con los fechamiento de los complejos arquitectónicos circulares.

Ahora, si bien la ocupación del cañón Juchipila se nos presenta como una cronología larga, durante el Posclásico aparentemente no es igual a la que se da en épocas previas. De hecho, sabemos algo más de ella por la información que dan las fuentes históricas (aunque en muchos casos sea contradictoria en cuanto al modo de vida de los grupos), es así como sabemos que durante la época de la conquista y la guerra del Mixtón esta región estuvo habitada por los caxcanes (Ruiz Medrano 1994).

De igual forma, si observamos la distribución de sitios en la región veremos que su distribución corresponde de forma obvia al acceso a áreas de captación producidas por el Río Juchipila y los valles fértiles que se formaron entre ellas. De esta manera, las condiciones biogeográficas estuvieron entre los factores claves para el asentamiento de grupos humanos en la zona.

Las condiciones dadas en este corredor natural fueron de vital importancia para funcionar como ruta de interacción entre las sociedades que habitaron la región, ya que les permitía una ruta con acceso a recursos y poblaciones, aunque esto no implique en algún momento que hubiese también cierto grado de rechazo a algún grupo o individuos en particular, como le aconteció a los nuevos colonos. Esta ruta era poco agreste biogeográficamente a diferencia de las que rodean la zona y la presencia de asentamientos nos hace suponer que ésta fue la condición en esta parte de Mesoamérica.

No creo necesario hacer mayor énfasis en que las rutas prehispánicas no eran las más indicadas para el paso de caballos, recuas y ca- 
rretas, ¿por qué habrían de estarlo? Este hecho fue considerado como un problema por los nuevos colonos en su primer intento de Camino Real. En el mejor de los casos, los caminos pudieron haber tenido una función simbólica y ritual, además de la funcional, pero definitivamente jamás se pensaron para el paso de animales de transportación y/o tiro. Los medios de transporte eran diferentes así como la cantidad de objetos trasportados por las rutas. Es bien sabido que estas rutas, caminos y veredas fueron lo suficientemente buenas para satisfacer los requerimientos de transporte de los nativos, así que no tenían porque estar invirtiendo tiempo y esfuerzo en la construcción y mantenimiento de caminos como se hicieron en sociedades occidentales y como lo hicieron los espańoles en los caminos reales. Muchas fueron utilizadas y transformadas más tarde en caminos, muchas otras semiabandonadas y otras tantas olvidadas (Calvo 1997).

La tecnología para la construcción de caminos para carretas y vagones respondía a nuevos requerimientos de transportación. En el caso de aquellos que pasaban por los valles que se forman en el Cañón de Juchipila, la forma de transporte a pie ofrecía una ruta de tráfico multidireccional y la posibilidad de crear alternativas cuando una de las establecidas no permitía su libre tránsito. Sin embargo, las condiciones del terreno no fueron las más indicadas para el paso de la caballería y carretas (sin importar que tan rápidas pudieran ser y que tanto más pudieran cargar). Por otra parte, la existencia de Guadalajara y Zacatecas fue lo que permitió la consolidación de grupos de resistencia entre las poblaciones indígenas locales que dieron como resultado las muchas escaramuzas que eventualmente llevaron a la Guerra del Mixtón y la Guerra Chichimeca. Considero innecesario recalcar que las principales revueltas indígenas se produjeron como respuesta a las políticas de expansión y, por tanto, fue necesario considerar la creación de caminos más seguros. Los ataques, los saqueos y los asesinatos a las caravanas que usaban los caminos obligaron al virreinato a crear fuerzas militares para proteger estos caminos, sin embargo, la incapacidad de las guardias para detener los embates de los chichimecas fue lo que llevó a las autoridades a tomar otras rutas más seguras, como sucedió con las de 


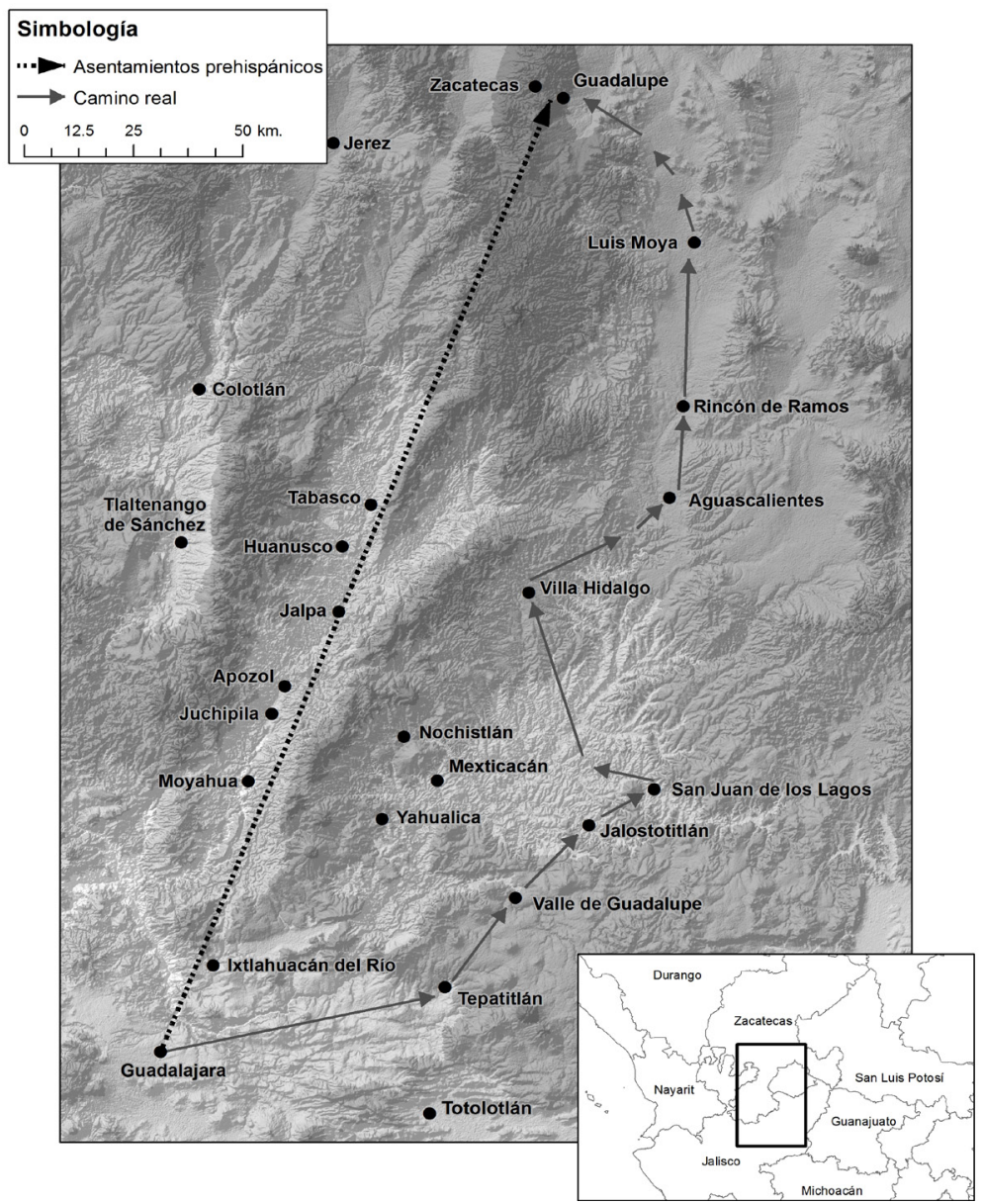

Camino Real

Palmillas-Zacatecas

Cuicillos-Palmillas

Tepezalá-Cuicillos

Aguascalientes-Tepezalá

Ciénega de Portugal-

Aguascalientes

Paso de carretas-Ciénega

de Portugal

Teocaltiche-Paso de Carretas

Paso del Sabino-Teocaltiche

Mitic-Paso de Sabino
Paso de La Laja-Mitic: Opción 1) La Venta-Paso de La Laja $=17 \mathrm{~km}$

Paso de la Laja $=22 \mathrm{~km}$

Opción 2) La Venta-

Jalostotitlan $=25 \mathrm{~km}$

Jalostotitlan-Mitic $=14 \mathrm{~km}$

La Venta-Paso de La Laja

Tepatitlán-La Venta

Puente Grande-Tepatitlán

Guadalajara-Puente Grande
Asentamientos PREHISPÁNICOS Tabasco

Huanusco

Jalpa

Apozol

Juchipila

Moyahua

Yahualica

Ixtlahuacan del Río

Elaborado por M. Nicolás Caretta con el apoyo de Marco Antonio Hernández de la Unidad de Cómputo de Colmich. 
Michoacán a Guadalajara en lugar de tomar la ruta del Camino Real de México a Guadalajara para llegar a Zacatecas. Esta situación de resistencia fue la que se enfrentó en la región del Cañón de Juchipila, la ruta más directa entre Guadalajara y Zacatecas y su primer intento de Camino Real.

Presento aquí, pues, un mapa donde podemos ver la distribución de los asentamientos prehispánicos presumiblemente del Posclásico que se encuentra en esa área del Cañón y que conecta a la región con el resto de centro-norte del país y, a su vez, es la ruta natural hacia la parte de Zacatecas y Durango. Esta ruta interior eventualmente continuaría hacía el norte hasta alcanzar Casas Grandes y otros sitios ubicados en latitudes más norteñas.

\section{BibliografíA}

Acuña, René, Relaciones Geográficas del Siglo XVI: Nueva Galicia, México, unam, 1988.

Anónimo, Relación de la Conquista de Nueva Galicia, alzose año de 1542. Anónima Tercera del Instituto Jalisciense de Antropología e Historia, en Crónicas de la Conquista del Reino de Nueva Galicia en Territorio de la Nueva España, Guadalajara, H. Ayuntamiento de la Ciudad de Guadalajara, Instituto Jalisciense de Antropología e Historia, INAH, México, 1963a, 317-343.

Anónimo, Relación de la Jornada que hizo Nuño de Guzmán a Nueva Galicia. Primera Relación, en Crónicas de la Conquista del Reino de Nueva Galicia en Territorio de la Nueva España, Guadalajara, H. Ayuntamiento de la Ciudad de Guadalajara, Instituto Jaliciense de Antropología e Historia, INAH, México, 1963b, 285-314.

Arlegui, José, Crónicas de la Provincia de N.S.P.S. Francisco de Zacatecas, México, Cumplido (original impreso por Bernardo Hogal), 1851 [1737].

Bakewell, P. J., Minería y sociedad en el México colonial, Zacatecas (1546-1700), México, FCE, 1997.

Baus de Czitrom, Carolyn, Tecuexes y Cocas. Dos grupos de la región de Jalisco en el siglo XVI, México, INAH, 1982. 
, "The Tecuexes: Ethnohistory and Archaeology", en The Archaeology of West and Northwest Mesoamerica, M. Foster y Phil Weigand, eds., Boulder, Westview Press, 1985, 93-117.

BeLl, Betty, "Excavations at El Cerro Encantado, Jalisco", en Betty Bell, ed., The Archaeology of West Mexico, Ajijic, West Society for Advanced Study, 1974, 147-167.

Benítez, José R., "Los Fundadores”, en José María Muría y Jaime Olveda, comps., Generalidades históricas sobre la fundación de Guadalajara. Lecturas históricas de Guadalajara I, Guadalajara, INAH-UDG, 1991 [1941].

Braniff, Beatriz, "Secuencias arqueológicas en Guanajuato y Cuenca de México: Intento de correlación”, en Teotihuacan, XI Mesa Redonda, México, sMA, 1972, 2, 273-232.

, Morales, Guanajuato, y la tradición Chupicuaro, Colección Científica, Serie Arqueología, México, INAH, 1998.

Cabrero, María Teresa y Carlos López, "Hallazgo de una tumba sellada en el Cañón de Bolaños, Jalisco", Antropológicas, núm. 1, México, unam, 1993, 1-27.

Calvo, Thomas, Por los caminos de la Nueva Galicia: Transportes y transportistas en el siglo XVI, Guadalajara, UDG-Cemca, 1997.

Crónicas de la Conquista del Reino de Nueva Galicia en Territorio de la Nueva España, Guadalajara, H. Ayuntamiento de la Ciudad de Guadalajara, Instituto Jalisciense de Antropología e Historia, INAH, México, 1963.

Chávez Haynoe, Arturo, "Las Comunicaciones", en José María Muría y Jaime Olveda, comps., Generalidades históricas sobre la fundación de Guadalajara. Lecturas históricas de Guadalajara I, Guadalajara, INAH-UDG, 1991 [1943].

Chávez Hayhoe, Arturo, “Segunda Fundación”, en José María Muría y Jaime Olveda, comps., Generalidades históricas sobre la fundación de Guadalajara. Lecturas históricas de Guadalajara I, Guadalajara, INAH-UDG, 1991 [1953].

De los Reyes, Aurelio, Los caminos de la plata, México, $1^{\text {a }}$ edición, Gobierno del Estado de Zacatecas, Patronato de Minería Cinco Siglos en México, Universidad Iberoamericana, 1991.

FÁbregas Puig, Andrés, La formación histórica de una región: Los 
Altos de Jalisco, Colección Miguel Otón de Mendizábal núm. 5, Ciesas, 1986.

Gerhard, Peter, La frontera norte de la Nueva España, México, UNAM, 1996.

GaLVÁN, Javier, Rescate arqueológico en el fraccionamiento tabachines, Zapopan, Jalisco, Cuadernos de los Centros 28, México, Dirección de los Centros Regionales INAH, 1976. , Las tumbas de tiro del valle de Atemajac, Jalisco, Colección Científica, Serie Arqueología 239, México, INAH, 1991.

Gómez Gastélum, L., y R. A. De la Torre, "Figurillas Cerrito de García de la cuenca de Sayula, Jalisco", Estudios del Hombre 3, Guadalajara, Universidad de Guadalajara, 1996, 127-150.

Kelley, Charles, "Archaeology of the Northern Frontier: Zacatecas and Durango", en Gordon F. Ekholm e Ignacio Bernal, eds., Handbook of Middle American Indians, vol. 11, Archaeology of Northern Mesoamerica, Austin, The University of Texas Press, 1971, 768-804.

JimÉnez BetTs, Peter F., "Ciertas inferencias de la arqueología del Sur de Zacatecas" en R. Brambila y A. Crespo, eds., Primera Reunión sobre Sociedades Prehispánicas en el Centro Occidente de México: Memoria, México, Centro Regional de Querétaro, Cuadernos de Trabajo I, INAH, 1988.

, "Perspectivas sobre la arqueología de Zacatecas" en Arqueología 5, Dirección de Monumentos Prehispánicos, México, INAH, 1989, 7-50.

Jiménez Betts, Peter F. y J. Andrew Darling, "Archaeology of Southern Zacatecas: The Malpaso, Juchipila, and ValparaisoBolaños Valleys", en Greater Mesoamerica: The Archaeology of West and Northern Mexico, Salt Lake City, The University of Utah Press, 2000, 155-180.

López Mestas, Lorenza y Jorge Ramos de la Vega, Investigaciones arqueológicas en la sierra de Comanja-Guanajuato, Tesis, México, Universidad Autónoma de Guadalajara, ENAH, 1992.

López Mestas, Lorenza et al. "Sitios y materiales: Avances del proyecto arqueológico Altos de Jalisco", en Eduardo Williams, ed., Contribuciones a la arqueología y etnohistória del occidente de 
México, Zamora, El Colegio de Michoacán, 1994, 279-296.

Medina, Humberto, El paisaje ritual del Valle de Malpaso, Tesis de Licenciatura, México, ENAH, 2000.

Meighan, C. W., ed., “The Archaeology of Amapa Nayarit”, Monumenta Archaeologica, 2, Los Angeles, The Institute of Archaeology, University of California, 1976.

Mота y Escobar, Alonso de la, Descripción geográfica de los reinos de la Nueva Galicia, Nueva Vizcaya y Nuevo León, México, México, Antigua Librería de Robledo, José Porrúa e Hijos, 1940.

Mota Padilla, Matías de la, Historia de la Nueva Galicia, Colección Histórica de Obras Facsimilares, Guadalajara, Instituto Jalisciense de Antropología, Universidad de Guadalajara, 1973 [1742].

Nicolás Caretta, M., Proyecto Arqueológico Cerro de Las Ventanas, Juchipila, Zacatecas, México, México, Archivo de Monumentos Prehispánicos, INAH, 2002.

, "The Route to Cibola: The Prehispanic Routes and the Colonial Roads of the Nueva Galicia”, VIII Coloquio del Camino Real de Tierra Adentro, Albuquerque, Nuevo Mexico, octubre, 2003.

Nicolás Caretta, M. y Antonio Motilla, “¿Fuera o dentro de Mesoamérica? Sociedades y territorialidad en el centro norte de México en el siglo xvI", Tiempo y Región: Estudios Históricos y Sociales, Querétaro, U. Querétaro-INAH, 2008, 335-348.

Noyola, A., "Análisis preliminar de la cerámica del fraccionamiento San Juan Atoyac, Jalisco", en Eduardo Williams, ed., Contribuciones a la arqueología y etnohistoria del occidente de México, Zamora, El Colegio de Michoacán, 1994, 55-92.

PÁez Brotchie, Luis, "La Primera Guadalajara (Nochistlán)”, en José María Muría y Jaime Olveda, comps., Generalidades históricas sobre la fundación de Guadalajara. Lecturas históricas de Guadalajara I, Guadalajara, INAH-UDG, 1991 [1961].

, "La Segunda y la Tercera Guadalajara”, en José María Muría y Jaime Olveda, comps., Generalidades históricas sobre la fundación de Guadalajara. Lecturas históricas de Guadalajara I, Guadalajara, INAH-UDG, 1991 [1961]. 
Powell, Philip, La Guerra Chichimeca (1550-1600), México, FCE, 1984.

Capitán mestizo: Miguel Caldera y la frontera norteña. La pacificación de los Chichimecas (1548-1597), México, FCE, 1987.

Ramírez Urrea, Susana, "El papel interregional de la cuenca de Sayula, Jalisco, en el Epiclásico y Postclásico temprano: Observaciones preliminares", Conferencia IV Coloquio Bosch-Gimpera, Guadalajara, Jalisco, 1997.

Ruiz Medrano, Ethelia, "Versiones sobre un fenómenos rebelde. La guerra del Mixtón en Nueva Galicia”, en Contribuciones a la arqueología y etnohistoria del Occidente de México, Zamora, Colegio de Michoacán, 1994.

Schöndube, Otto, "Época prehispánica”, en José María Muría, Historia de Jalisco: Vol. 1, Desde los tiempos prehistóricos hasta fines del siglo XVII, Guadalajara, Unidad Editorial del Gobierno de Jalisco, 1980, 113-257.

, "El Occidente de México hasta la época tolteca", en Los orígenes de México, México, Ediciones Salvat Mexicana, 1982, 271-298.

Schöndube, O. y J. Galván V., "Salvage Archaeology at El GrilloTabachines, Zapopán, Jalisco, México”, en C. L. Riley y B. C. Hedrick, eds., Across the Chichimec Sea: Papers in Honor of J. Charles Kelley, Carbondale, Southern Illinois University Press, 1978, 144-164.

Tello, Antonio, Crónica Miscelánea de la Sancta Provincia de Xalisco, vols. I, II, III, México, Gobierno del Estado de Jalisco, UDG, INAH, 1984.

Trombold, Charles, Ancient Roads Networks and Settlements Hierarchies in the New World, New Directions in Archaeology, Cambridge, Cambridge University Press, 1991.

Ulloa Nieto, Sergio, El camino más largo. Historia del Camino Real Guadalajara-Zacatecas en el Siglo XVI, Tesis de Licenciatura, México, ENAH, 2002.

Weigand, Phil, "Evidence for Complex Societies during Western Mesoamerican Classic Period", en Michael Foster y Phil Wei- 
gand, eds., The Archaeology of West and Northwest Mesoamerica, Boulder, Westview Press, 1985, 47-92.

Weigand, Phil, A. García de Weigand y J. A. Darling, "El sitio arqueológico: Cerro de Tepisuazco (Jalpa, Zacatecas) y sus relaciones con la tradición Teuchitlán”, en C. González Pérez, ed., Tercer simposium: Los Altos de Jalisco a fin de siglo, Guadalajara, Sistema de Educación Media Superior, Universidad Autónoma de Guadalajara, 1999, 241-274.

FECHA DE RECEPCIÓN DEL ARTículo: 27 de enero de 2010

FECHA DE ACEPTACIÓN Y RECEPCIÓN DE LA VERSIÓN FINAL: 14 de marzo de 2011 